\title{
Model of a Two-Dimensional Marginal Fermi Liquid Superconductor
}

\author{
Yu-Liang Liu and T. K. Ng \\ Department of Physics, Hong Kong University of Science and Technology, Clear Water Bay Road, Kowloon, \\ Hong Kong, People's Republic of China
}

(Received 2 July 1999)

\begin{abstract}
In this paper we study a model of an $s$-wave marginal Fermi liquid superconductor in two dimensions. Besides the usual Bardeen-Cooper-Schrieffer (BCS) point attractive interaction, the fermions in our system also interact via a long-range repulsive interaction $v(q)=g_{D} / q^{2}$. We find that although superconductivity is not destroyed by the long-ranged repulsive interaction (demonstrated by the response to electromagnetic magnetic fields), the BCS pairing function $\Delta_{\mathrm{BCS}}$ is strongly suppressed, resulting in a power-law correlation $\left\langle\Delta_{\mathrm{BCS}}(\vec{x}) \Delta_{\mathrm{BCS}}(0)\right\rangle \sim|\vec{x}|^{-2 \gamma}$. This observation is explained within a general picture of the marginal Fermi liquid.
\end{abstract}

PACS numbers: 71.10.Hf, 74.20.-z, 74.25.-q

Since the discovery of high- $T_{c}$ superconductors, there has been enormous interest in the study of non-Fermi liquid theories in two dimensions [1-3]. The normal state of high- $T_{c}$ superconductors shows rich physical behavior which seems to deviate from usual Landau-Fermi liquid theory predictions. Although there exist many theoretical studies on microscopic theories of non-Fermi liquid normal states, at present there exist very few systematic investigations into how the superconducting state is affected by non-Fermi liquid behavior.

In this paper we study a microscopic model of an $s$-wave marginal Fermi liquid superconductor at two dimensions. The Hamiltonian of our model consists of two parts, $H=$ $H_{\mathrm{mfl}}+H_{\mathrm{BCS}}$, where

$$
\begin{aligned}
H_{\mathrm{mf} 1}= & \sum_{\vec{k}, \sigma} \epsilon(k) \psi_{\sigma}^{\dagger}(\vec{k}) \psi_{\sigma}(\vec{k}) \\
& +\frac{1}{2 L^{2}} \sum_{|\vec{q}| \leq q_{c}} v(q) \rho(\vec{q}) \rho(-\vec{q})
\end{aligned}
$$

and

$$
\begin{aligned}
H_{\mathrm{BCS}}=-\int d^{2} x\{ & {\left[\Delta(\vec{x}, t) \psi_{\downarrow}^{\dagger}(\vec{x}, t) \psi_{\uparrow}^{\dagger}(\vec{x}, t)+\text { H.c. }\right] } \\
& \left.-\frac{1}{U}|\Delta(\vec{x}, t)|^{2}\right\}
\end{aligned}
$$

where $\epsilon(k)=\frac{k^{2}}{2 m}-\mu, \rho(\vec{x})=\sum_{\sigma} \psi_{\sigma}^{\dagger}(\vec{x}) \psi_{\sigma}(\vec{x})$ is the electron density operator, $\psi_{\sigma}(\vec{x})\left[\psi_{\sigma}^{\dagger}(\vec{x})\right]$ are electronic annihilation (creation) operators with spin $\sigma=\uparrow, \downarrow$, and $L^{2}$ is the volume of the system. $q_{c}$ is a high momentum cutoff for the interaction potential $v(q)=g_{D} / q^{2}$. We assume $q_{c} \ll k_{F}$ in the following so that a systematic expansion of the problem in powers of $q_{c} / k_{F}$ is possible. $\Delta(\vec{x}, t)\left[\Delta^{*}(\vec{x}, t)\right]$ are Hubbard-Stratonovich (HS) fields introduced to decouple the (attractive) electronelectron interaction $U . U$ is a coupling constant of an unknown mechanism which leads to electron pairing. Note that in the usual BCS mean-field theory, $\Delta(\vec{x}, t) \rightarrow$
$\Delta_{\mathrm{BCS}}=-U\left\langle\psi_{\uparrow}(\vec{x}) \psi_{\downarrow}(\vec{x})\right\rangle$, where $\Delta_{\mathrm{BCS}}$ is the mean-field BCS gap. The repulsive interaction potential $v(q)$ among the electrons may originate from a U(1) gauge field such as in a $t$ - $J$ model $[3,4]$, or a vortex-vortex interaction where each vortex is combined to one electron [5].

The Hamiltonian $H_{\mathrm{mfl}}$ has been carefully studied [6] where it is known that it describes a state with Fermiliquid-like particle-hole and collective excitation spectrums, but with nevertheless a vanishing Fermi surface discontinuity $z \rightarrow 0$ [6] because of the orthogonality catastrophe effect associated with induced long-ranged density fluctuations when an additional electron is introduced [6] (marginal Fermi liquid behavior). Notice that in the absence of the interaction term $v(q)$, the system would be a regular $s$-wave superconductor at two dimensions, described by a constant pairing function $\Delta_{\mathrm{BCS}}$ in mean-field theory. Here we examine whether superconductivity is destroyed by the marginal Fermi liquid behavior, and, if it survives, whether and how its behavior will be modified. Note that even though it is believed that in the high- $T_{c}$ cuprates the electron pairing function has a $d$-wave symmetry, interesting conclusions may still be drawn from studying this simple model as we see in the following.

To solve this model Hamiltonian we first introduce another Hubbard-Stratonovich field $\phi(q, t)$ to decouple the repulsive electron-electron interaction. In terms of $\phi$ and $\Delta$ fields, the Lagrangian of the system $L$ can be written as

$$
\begin{aligned}
L=i \int_{-T}^{T} d t[ & \frac{1}{L^{2}} \sum_{|\vec{q}| \leq q_{c}} \frac{\phi(\vec{q}, t) \phi(-\vec{q}, t)}{2 v(q)} \\
& \left.+\int d^{2} x \Psi^{\dagger}(\vec{x}, t) \hat{G}^{-1}(\vec{x}, t,[\phi]) \Psi(\vec{x}, t)\right],
\end{aligned}
$$

where $T \rightarrow \infty$, and

$$
\begin{aligned}
\hat{G}^{-1}(\vec{x}, t,[\phi])= & i \partial_{t}+\left(\frac{\nabla^{2}}{2 m}+\mu-\phi(\vec{x}, t)\right) \sigma^{z} \\
& -\tilde{\Delta}(\vec{x}, t),
\end{aligned}
$$


where $\sigma^{z}$ is a Pauli matrix,

$$
\Psi(\vec{x}, t)=\left(\begin{array}{c}
\psi_{\uparrow}(\vec{x}, t) \\
\psi_{\downarrow}^{\dagger}(\vec{x}, t)
\end{array}\right)
$$

is a Nambu spinor, and

$$
\tilde{\Delta}(\vec{x}, t)=\left(\begin{array}{cc}
0, & \Delta(\vec{x}, t) \\
\Delta^{*}(\vec{x}, t), & 0
\end{array}\right) .
$$

Note that the Lagrangian is now quadratic in electron fields after the HS bosonic fields $\Delta(\vec{x}, t)$ and $\phi(\vec{x}, t)$ are introduced.

We solve the problem in two steps: (i) For each configuration of $\phi(\vec{x}, t)$ field, we first solve the self-consistent BCS mean-field equations

$$
\begin{aligned}
\hat{G}^{-1}(\vec{x}, t,[\phi]) G\left(\vec{x}, \vec{x}^{\prime}, t, t^{\prime},[\phi]\right)= & \delta\left(\vec{x}-\vec{x}^{\prime}\right) \\
& \times \delta\left(t-t^{\prime}\right), \\
\Delta(\vec{x}, t ;[\phi])= & -U\left\langle\psi_{\uparrow}(\vec{x}, t ;[\phi])\right. \\
& \left.\times \psi_{\downarrow}(\vec{x}, t ;[\phi])\right\rangle_{0},
\end{aligned}
$$

approximately using an eikonal expansion $[7,8]$ or functional bosonization [9] approach, where $\langle A\rangle$ is the expectation value of operator $A$ at fixed configuration $\phi(\vec{x}, t)$ (ii) With the approximate Green's function $G\left(\vec{x}, \vec{x}^{\prime}, t, t^{\prime} ;[\phi]\right)$ obtained for each configuration $\phi(\vec{x}, t)$, we can construct an approximate action $S_{\text {eff }}[\phi]$ in terms of the $\phi(\vec{x}, t)$ field in the eikonal expansion. The ground state expression value $\langle G(\vec{x}, t)\rangle$ and other higher-order correlation functions can be evaluated as

$$
\begin{aligned}
\left\langle G\left(\vec{x}-\vec{x}^{\prime}, t-t^{\prime}\right)\right\rangle & =\left\langle G\left(\vec{x}, \vec{x}^{\prime}, t, t^{\prime} ;[\phi]\right\rangle_{\phi}\right. \\
& =\frac{\int d \phi G\left(\vec{x}, \vec{x}^{\prime}, t, t^{\prime} ;[\phi]\right) e^{-S_{\text {eff }}[\phi]}}{\int d \phi e^{-S_{\text {eff }}[\phi]}},
\end{aligned}
$$

etc. Notice that in one dimension the eikonal expansion or functional bosonization method can be viewed as alternate approaches to the usual bosonization procedure and can reproduce the same result as the usual bosonization method [7-9]. At higher dimensions, the method treats only the forward scattering amplitude at small wave vector $q$ exactly but can be used as a systematic expansion technique in powers of $q_{c} / k_{F}[7,8]$ for fermions interacting with scalar potential $v(q)$ where the forward scattering amplitude is dominating. In our present case, the marginal Fermi liquid behavior comes from singular coupling of fermions to plasmons (density fluctuations) at small wave vector $q \rightarrow 0$ [6] and this behavior can be recovered correctly to leading order in eikonal expansion $[7,8,10]$. The new ingredient we introduce here is to include the BCS mean-field theory in a self-consistent way into the formalism. In the following we shall give some brief mathematical outlines on how the eikonal expansion is carried out. First we review the case when the BCS term is absent (normal marginal Fermi liquid).

In this case the matrix Green's function $G\left(\vec{x}, \vec{x}^{\prime}, t, t^{\prime} ;[\phi]\right)$ becomes a number function. In the eikonal expansion, we write $G$ as $[9,10]$

$$
\begin{aligned}
G\left(\vec{x}, \vec{x}^{\prime}, t, t^{\prime} ;[\phi]\right)= & \frac{1}{T L^{2}} \sum_{\vec{k}, \omega} g_{0}(\vec{k}, \omega) e^{-i\left[\omega t-\vec{k} \cdot \vec{x}+Q_{\vec{k}}(\vec{x}, t ;[\phi])\right]} \\
& \times e^{i\left[\omega t^{\prime}-\vec{k} \cdot \vec{x}^{\prime}+Q_{\vec{k}}\left(\vec{x}^{\prime}, t^{\prime} ;[\phi]\right)\right]}
\end{aligned}
$$

where $g_{0}(\vec{k}, \omega)=[\omega-\epsilon(k)]^{-1}, \epsilon(k)=k^{2} /(2 m)-\mu$, and the function $Q_{\vec{k}}(\vec{x}, t ;[\phi])$ is obtained approximately in the eikonal expansion. To leading order in $q_{c} / k_{F}$ we obtain $[8,10]$

$$
Q_{\vec{k}}(\vec{x}, t ;[\phi])=-\frac{1}{T L^{2}} \sum_{|\vec{q}|<q_{c}, \omega} e^{i(\omega t-\vec{q} \cdot \vec{x})} \frac{\phi(\vec{q}, \omega)}{\omega-\frac{\vec{k} \cdot \vec{q}}{m}} .
$$

Physically, the $e^{-i Q_{\vec{k}}(\vec{x}, t ;[\phi])}$ term describes the dressing of the fermions by density fluctuations $\phi$. This effect is treated nonperturbatively in eikonal expansion where an infinite series of terms in $\phi$ is summed up to an exponential form. For the special case of a $1 / q^{2}$ potential in two dimensions, it turns out that the infrared singularity which leads to marginal Fermi liquid behavior comes from plasma oscillations with energy $\omega \sim \Omega_{0}=\sqrt{g_{D} \rho_{0} / m}$, where $\rho_{0}$ is the electron density [6,10]. As a result one may neglect the $\vec{q} \cdot \vec{k} / m$ term in $Q_{\vec{k}}$ as far as the leading infrared singularity is concerned, and we obtain

$$
G\left(\vec{x}, \vec{x}^{\prime}, t, t^{\prime} ;[\phi]\right)=G_{0}\left(\vec{x}-\vec{x}^{\prime}, t-t^{\prime}\right) e^{i\left[Q(\vec{x}, t)-Q\left(\vec{x}^{\prime}, t^{\prime}\right)\right]},
$$

where $Q(\vec{x}, t) \sim-\frac{1}{T l^{2}} \sum_{\vec{q}, \omega} e^{i(\omega t-\vec{q} \cdot \vec{x})} \frac{\phi(\vec{q}, \omega)}{\omega} ; G_{0}(\vec{x}, t)$ is the usual free fermion Green's function. An eikonal expansion can also be performed for the effective action $S_{\text {eff }}(\phi)$. To leading order in $q_{c} / k_{F}, S_{\text {eff }}[\phi]$ is just the RPA effective action

$$
S_{\mathrm{eff}}[\phi]=\frac{1}{2 T L^{2}} \sum_{q<q_{c}, \omega}|\phi(\vec{q}, \omega)|^{2}\left[v(q)^{-1}-\chi_{0}(q, \omega)\right],
$$

where $\chi_{0}(q, \omega)$ is the usual Lindhard function. This result can be understood by noting that (i) higher-order terms in power of $\phi$ involve sums over extra momentum $q<q_{c}$ and generate higher powers in $q_{c} / k_{F}$ and (ii) the singular behavior at small $q$ which is present in a quadratic term will be absent in higher-order terms. Notice also that the marginal Fermi liquid behavior originates from electronplasmon coupling in the present model [6]. Therefore, as far as the marginal Fermi liquid behavior is concerned, the effective action may be further simplified by keeping only the plasma-pole contributions [6] to $S_{\text {eff }}[\phi]$. This is in contrast to many other models of marginal Fermi liquids where the marginal Fermi liquid behavior is generated from coupling of electrons to anomalous low energy particle-hole excitations [2,3]. With Eqs. (4) and (6a) and (6b), the one-particle Green's function for $H_{\mathrm{mfl}}$ can be computed easily and the result is in agreement with the previous result obtained by Bare and Wen $[6,10]$.

With Eq. (6a), it is easy to show that the corresponding matrix Green's function in the presence of the BCS attractive interaction which keeps the leading infrared singularity has the form 


$$
G\left(\vec{x}, \vec{x}^{\prime}, t, t^{\prime} ;[\phi]\right)=\left(\begin{array}{cc}
G_{0}^{\mathrm{BCS}} e^{i\left[Q(\vec{x}, t)-Q\left(\vec{x}^{\prime}, t^{\prime}\right)\right]}, & F_{0}^{\mathrm{BCS}} e^{i\left[Q(\vec{x}, t)+Q\left(\vec{x}^{\prime}, t^{\prime}\right)\right]} \\
F_{0}^{\mathrm{BCS} *} e^{-i\left[Q(\vec{x}, t)+Q\left(\vec{x}^{\prime}, t^{\prime}\right)\right]}, & G_{0}^{\mathrm{BCS} *} e^{-i\left[Q(\vec{x}, t)-Q\left(\vec{x}^{\prime}, t^{\prime}\right)\right]}
\end{array}\right),
$$

and with corresponding pairing function

$$
\Delta(\vec{x}, t ;[\phi])=\frac{U \Delta}{2 L^{2}} \sum_{\vec{k}} \frac{1}{E(k)} e^{-2 i Q(\vec{x}, t)},
$$

where $G_{0}^{\mathrm{BCS}} \equiv G_{0}^{\mathrm{BCS}}\left(\vec{x}-\vec{x}^{\prime}, t-t^{\prime}\right) \quad$ and $\quad F_{0}^{\mathrm{BCS}} \equiv$ $F_{0}^{\mathrm{BCS}}\left(\vec{x}-\vec{x}^{\prime}, t-t^{\prime}\right)$ are the usual BCS normal and anomalous Green's functions in the absence of the $\phi$ field, respectively, with the usual Fourier transform, $\quad G_{0}^{\mathrm{BCS}}(\vec{k}, i \omega)=\frac{i \omega+\epsilon(k)}{(i \omega)^{2}-E(k)^{2}} \quad$ and $\quad F_{0}^{\mathrm{BCS}}(\vec{k}, i \omega)=$ $\frac{-\Delta}{(i \omega)^{2}-E(k)^{2}}$, where $E(k)=\sqrt{\Delta^{2}+\epsilon(k)^{2}}$, and $\Delta$ is determined by the usual BCS mean-field equation $1=\frac{U}{2 L^{2}} \sum_{k} \frac{1}{E_{k}}$ [see (7b)]. We assume weak attractive interaction $U$ and $\Delta \ll \Omega_{0}$ in the following discussions.

As in the normal state, to leading order in $q_{c} / k_{F}$, the effective action $S_{\text {eff }}[\phi]$ of the system in the presence of the BCS attractive interaction is the quadratic action of BCS superconductors for scalar potentials,

$$
\begin{aligned}
S_{\mathrm{eff}}[\phi]= & \frac{1}{2 T L^{d}} \sum_{q \leq\left|q_{c}\right|, \Omega}\left[\frac{1}{v(q)}-\chi_{\mathrm{BCS}}(q, \Omega)\right] \\
& \times|\phi(q, \Omega)|^{2},
\end{aligned}
$$

where $\chi_{\mathrm{BCS}}(q, \Omega)$ is the irreducible density-density response function (counterpart of Lindhard function) in BCS theory. In the long wavelength limit $q \rightarrow 0, \Omega \gg k_{F} q / m$, $\chi_{\mathrm{BCS}}(q, \Omega)$ reduced to $\chi_{\mathrm{BCS}}(q \rightarrow 0, \Omega) \simeq \frac{\rho_{0}}{2 m}\left(\frac{q}{\Omega}\right)^{2}$. For a $v(q)=g_{D} / q^{2}$ potential, the effective action becomes in this limit

$$
S_{\mathrm{eff}}[\phi]=\frac{1}{2 T L^{2}} \sum_{q, \Omega} \frac{1}{v(q)}\left(1-\frac{\Omega_{0}^{2}}{\Omega^{2}}\right)|\phi(q, \Omega)|^{2},
$$

where $\Omega_{0}=\sqrt{g_{D} \rho_{0} / m}$ is the plasma frequency. We note that as far as plasma oscillation is concerned the effective action $S_{\text {eff }}[\phi]$ is unaffected by the presence of superconductivity. This is a direct manifestation of Higg's mechanism.

Using the effective action (9), we can calculate the thermodynamical average and correlation function of the pairing function and the normal and anomalous Green's functions by using their expressions in (7a) and (7b), respectively. We obtain

$$
\left\langle Q(\vec{x}, t) Q\left(\vec{x}^{\prime}, t^{\prime}\right)\right\rangle_{\phi} \simeq \begin{cases}-\frac{\gamma}{4} \ln \left|\vec{x}-\vec{x}^{\prime}\right|, & \left|t-t^{\prime}\right| \ll \frac{1}{\Omega_{0}}, \\ \frac{\gamma}{4} \ln \left(\frac{1}{q_{c} L}\right), & \left|t-t^{\prime}\right| \gg \frac{1}{\Omega_{0}},\end{cases}
$$

where $\gamma=\frac{g_{D}}{2 \pi \Omega_{0}}$, and, correspondingly,

$$
\begin{aligned}
\Delta_{\mathrm{BCS}}=\langle\Delta(\vec{x}, t,[\phi])\rangle_{\phi} & \simeq \Delta\left(\frac{1}{q_{c} L}\right)^{\gamma}, \\
\left\langle\Delta(\vec{x}, t,[\phi]) \Delta^{*}\left(\vec{x}^{\prime}, t,[\phi]\right)\right\rangle_{\phi} & \simeq \Delta^{2}\left(\frac{1}{\left|\vec{x}-\vec{x}^{\prime}\right|}\right)^{2 \gamma}, \\
\left\langle\Delta(\vec{x}, t,[\phi]) \Delta^{*}\left(\vec{x}^{\prime}, t^{\prime},[\phi]\right)\right\rangle_{\phi} & \simeq \Delta^{2}\left(\frac{1}{q_{c} L}\right)^{2 \gamma}, \quad\left|t-t^{\prime}\right| \rightarrow \infty .
\end{aligned}
$$

Notice that as the size of the system $L^{2} \rightarrow \infty$, the pairing function $\Delta_{\mathrm{BCS}}$ goes to zero because of the long-range interaction potential $v(q)$, and its (equal-time) correlation function shows a power-law behavior. The normal electron Green's function can also be calculated similarly. We obtain

$$
G\left(\vec{x}-\vec{x}^{\prime}, t-t^{\prime}\right) \simeq G_{0}^{\mathrm{BCS}}\left(\vec{x}-\vec{x}^{\prime}, t-t^{\prime}\right) \begin{cases}\left(\frac{1}{\left|\vec{x}-\vec{x}^{\prime}\right|}\right)^{\gamma}, & t \sim t^{\prime}, \\ \left(\frac{1}{q_{c} L}\right)^{\gamma}, & \left|t-t^{\prime}\right| \gg \frac{1}{\Omega_{0}} .\end{cases}
$$

Notice that a similar expression of the one-electron Green's function is also obtained in the normal state with $G_{0}^{\mathrm{BCS}}$ replaced by the usual free fermion Green's function.

There are two effects to be noticed. First of all, we find that the BCS pairing function is strongly suppressed by the long-range interaction potential, which seems to indicate that superconductivity is destroyed. Second, the oneelectron Green's function is strongly suppressed by a term $\left(q_{c} L\right)^{-\gamma}$ at frequency $\omega<\Omega_{0}$, resulting in a pseudogaplike structure in the tunneling density of state for $\omega<\Omega_{0}$, both in the normal state [6] and the superconductivity state.
Physically, this pseudogaplike structure indicates that in the presence of long-range repulsive interaction it is essentially impossible to add (or subtract) an electron without creating density fluctuations in the system [6] (orthogonality catastrophe). Notice, however, that although the differences between superconducting and normal states in the normal and anomalous one-electron Green's functions are strongly suppressed by $v(q)$ and are hardly visible when $\Delta \ll \Omega_{0}$, nevertheless, an order parameter $\Delta$ determined by the BCS mean-field equation still exists. We look into 
the meaning of nonzero $\Delta$ more carefully by examining directly the electromagnetic response of the system in the following.

Following standard linear-response theory analysis, we write in the Coulomb gauge,

$$
\begin{aligned}
\vec{J}(\vec{x}, t)= & \int d^{2} x^{\prime} P\left(\vec{x}-\vec{x}^{\prime}, t-t^{\prime}\right) \vec{A}\left(\vec{x}^{\prime}, t^{\prime}\right) \\
& -\frac{\rho_{0}}{m} \vec{A}(\vec{x} \cdot t),
\end{aligned}
$$

where $\vec{A}(\vec{x}, t)$ is the external (transverse) gauge field and $\vec{J}(\vec{x}, t)$ is the corresponding induced current. The response function $P(\vec{x}, t)$ is given by

$$
\begin{aligned}
& P\left(\vec{x}-\vec{x}^{\prime}, t-t^{\prime}\right)=\left\langle P\left(\vec{x}, \vec{x}^{\prime}, t, t^{\prime} ;[\phi]\right)\right\rangle_{\phi}, \\
& P\left(\vec{x}, \vec{x}^{\prime}, t, t^{\prime} ;[\phi]\right)=\frac{-i}{m^{2}} \operatorname{Tr} {\left[\left(\nabla_{x} \cdot \nabla_{x^{\prime}}\right) G\left(\vec{x}, \vec{x}^{\prime}, t, t^{\prime} ;[\phi]\right)\right.} \\
&\left.\times G\left(\vec{x}^{\prime}, \vec{x}, t^{\prime}, t ;[\phi]\right)\right],
\end{aligned}
$$

where $\left(\nabla_{x} \cdot \nabla_{x^{\prime}}\right) G_{1} G_{2}=\left(\nabla_{x} G_{1}\right) \cdot\left(\nabla_{x^{\prime}} G_{2}\right)+\left(\nabla_{x^{\prime}} G_{1}\right) \cdot$ $\left(\nabla_{x} G_{2}\right)-\left(\nabla_{x} \cdot \nabla_{x^{\prime}} G_{1}\right) G_{2}-G_{1}\left(\nabla_{x} \cdot \nabla_{x^{\prime}} G_{2}\right)$. Using Eqs. (7a) and (7b) and the fact that $\nabla Q(\vec{x}, t)$ introduces an extra power of $q$ in $Q$ and is thus higher order in $q_{c} / k_{F}$, we find that to leading order in $q_{c} / k_{F}$ the $Q(\vec{x}, t)$ factor does not contribute and $P(\vec{x}, t)$ is given by the usual BCS expression; i.e., the electromagnetic response of our system is unaffected by the marginal Fermi liquid behavior and is the same as usual BCS superconductors. In particular, the Fourier transform $P(\vec{q} \rightarrow 0, \omega=0)$ vanishes when $\Delta \neq 0$ indicating that the system exhibits the usual Meissner effect.

The apparent contradiction between the existence of a Meissner effect and the vanishing of a BCS order parameter $\Delta_{\mathrm{BCS}} \rightarrow 0$ can be resolved in the marginal Fermi liquid picture if we assume that superconductivity is formed by pairing of quasiparticles with pairing amplitude $\Delta_{\mathrm{qp}}=$ $\Delta \neq 0$. The bare fermion pairing amplitude is related to $\Delta_{\mathrm{qp}}$ by the usual Fermi liquid relation $\Delta_{\mathrm{BCS}} \sim z \Delta_{\mathrm{qp}}$ which is nonzero in the cases of usual Fermi liquids where $z \neq 0$. However, $z \sim\left(q_{c} L\right)^{-\gamma}$ and vanishes in the $L \rightarrow \infty$ limit in our system and $\Delta_{\mathrm{BCS}} \rightarrow 0$. Nevertheless, the system is still a superconductor because quasiparticles are in a BCS superconducting state. Notice that this is a rather general result of a $(z \rightarrow 0)$ marginal Fermi liquid superconductor. In particular, the argument is completely independent of the symmetry of the superconducting order parameters. Notice that recent quantum Monte Carlo studies on a $t-J$ model have indicated that the pairing function of electrons $\Delta_{\text {BCS }}$ seems to vanish as the size of the system increases [11]. This result was interpreted as indicating the absence of superconductivity in a $t-J$ model [11]. Our finding here suggests an alternative possibility: superconductivity may still exist in a $t-J$ model if the pairing quasiparticles have vanishing wave function overlap with bare electrons in the system.

Summarizing, using the eikonal expansion, we studied in this paper the influence of long-range repulsive electron-electron interaction on an $s$-wave superconductor at two dimensions. We find that the bare electron BCS pairing function $\Delta_{\mathrm{BCS}}$ is strongly suppressed by the plasma oscillation, with, however, superconductivity (Meissner effect) kept intact. We interpret the system as a marginal Fermi liquid superconductor where the pairing quasiparticles have vanishing wave function overlap $z^{\frac{1}{2}}$ with bare electrons in the system. In particular, we point out that this situation may occur in the $t$ - $J$ model where the ( $d$-wave) bare electron pairing function seems to vanish when the size of a system increases [11]. Note that our theory is valid to leading order expansion in $q_{c} / k_{F}$. The marginal Fermi-liquid effect arises from $q \rightarrow 0$ plasma fluctuations in our model and is treated exactly in the leading order theory. In particular, $q_{c} \ll k_{F}$ is introduced as a formal mathematical tool to guarantee a systematic expansion. We expect that higher-order corrections or a large value of $q_{c} / k_{F}$ will not modify our results qualitatively if the singular behavior at $q \rightarrow 0$ is the only source of infrared divergence appearing in our system.

Experimentally, we point out that a pseudogaplike structure in both the normal and superconducting states appears in the electron tunneling density of states in our model, as a result of the vanishing wave function overlap $z \rightarrow 0$. These features are observable in tunneling and photoemission experiments. Nevertheless, the electromagnetic responses of our system are qualitatively the same as usual BCS superconductors. All these features are being observed in the underdoped regime of the high- $T_{c}$ superconductors. Thus although our model is too simple and is definitely not directly applicable to describe high- $T_{c}$ cuprates, the underlying physics where these features are produced in our model may still be relevant to the understanding of high- $T_{c}$ superconductors.

This work is supported by HKUGC through RGC Grant No. HKUST6124/98P.

[1] P. W. Anderson, Science 273, 1196 (1987); Phys. Rev. Lett. 71, 1220 (1993).

[2] C. M. Varma et al., Phys. Rev. Lett. 63, 1996 (1989); C. M. Varma, Phys. Rev. Lett. 75, 898 (1995).

[3] N. Nagaosa and P. A. Lee, Phys. Rev. Lett. 64, 2450 (1990); X. G. Wen and P. A. Lee, Phys. Rev. Lett. 76, 503 (1996).

[4] L. B. Ioffe and A. I. Larkin, Phys. Rev. B 39, 8988 (1989).

[5] L. Balents, M. P. A. Fisher, and C. Nayak, Int. J. Mod. Phys. B 12, 1033 (1998); cond-mat/9811236.

[6] P. A. Bare and X. G. Wen, Phys. Rev. B 48, 8636 (1993).

[7] C. Castellani, C. Di Castro, and W. Metzner, Phys. Rev. Lett. 72, 316 (1994); C. Castellani and C. Di Castro, Physica (Amsterdam) 235C-240C, 99 (1994).

[8] P. Kopietz and G. E. Castilla, Phys. Rev. Lett. 76, 4777 (1996); E. S. Fradkin, Nucl. Phys. 76, 588 (1966).

[9] D. K. K. Lee and Y. Chen, J. Phys. A 21, 4155 (1988).

[10] T. K. Ng (unpublished).

[11] C. T. Shih, Y. C. Chen, H. Q. Lin, and T. K. Lee, Phys. Rev. Lett. 81, 1294 (1998). 